\title{
Synthesis and catalytic application of novel binaphthyl-derived phosphorous ligands
}

\author{
Kathrin Junge, Bernhard Hagemann, Stephan Enthaler, Giulia Erre, and Matthias Beller* \\ Leibniz-Institut für Katalyse e.V. an der Universität Rostock, Albert-Einstein-Straße 29a, D- \\ 18059 Rostock, Germany \\ E-mail: matthias.beller@catalysis.de
}

\section{Dedicated to Prof. Dr. Lutz F. Tietze on the occasion of his $65^{\text {th }}$ birthday}

\begin{abstract}
We describe our recent achievements in the synthesis of chiral phosphorous ligands and their application in catalytic asymmetric hydrogenation reactions. Special attention is given to the development of novel binaphthophosphepine ligands. Starting from enantiomerically pure 1,1'binaphthol or 2,2'-dimethyl-1,1'-binaphthyl and the building blocks A or B, new chelating Pligands have been synthesized in one step in good to excellent yields (68-95\%). The resulting chiral bidentate phosphines, phosphites and phosphonites were tested in various asymmetric hydrogenation benchmark reactions. Interestingly, small structural changes within the ligands exhibit a significant influence on the enantioselectivity of the hydrogenation reactions.
\end{abstract}

Keywords: Asymmetric hydrogenation, homogeneous catalysis, chiral phosphines

\section{Introduction}

Catalysis, which is the science of accelerating chemical transformations, enables the chemical and pharmaceutical industry to offer a wide range of products for our health, environment, and nutrition. This makes catalysts indispensable for the needs of today's society and important tools for increasing sustainability. Catalysts are mainly used for environmental protection, the production of bulk and fine chemicals, oil processing in refineries, and polymer synthesis.

For some years our main area of scientific interest has been applied homogeneous catalysis with organometallic complexes. A key issue for controlling the activity, productivity, and selectivity of a given transition metal complex is the synthesis of molecularly defined catalytic centers. In other words, the electronic and steric properties of each active center can be controlled by using organic ligands, which coordinate to the metal center. Thus, "ligandtailoring" constitutes an extremely powerful tool to control all kinds of selectivity for a desired catalytic reaction and to influence catalyst stability and activity. Due to the advancements in organometallic chemistry and organic ligand synthesis, nowadays a plethora of ligands [P-, N-, 
and recently C-based ligands) is theoretically available ( $>>100.000)$. A selection of ligands recently developed in our laboratory is shown in Scheme 1. Apart from new aryl and alkyl phosphines 1-4, ${ }^{1}$ tridentate nitrogen ligands ${ }^{2} 5$ and carbenes have been synthesized in recent years. In particular the importance of carbenes ${ }^{3}$ is underlined by the fact that in 2005 the Nobel Prize in chemistry was awarded to Y. Chauvin, R. H. Grubbs and R. R. Schrock for the development of metathesis.

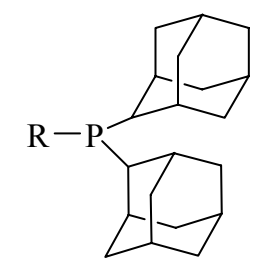

$\mathrm{R}=\mathrm{n}-\mathrm{Bu}, \mathrm{C}_{6} \mathrm{H}_{5} \mathrm{CH}_{2}, \mathrm{i}-\mathrm{Pr}$

1<smiles>[Y19]Cc1[X]c2ccccc2n1[14CH3]</smiles>

$\mathrm{X}=\mathrm{N} ; \mathrm{CH}_{2}$

4

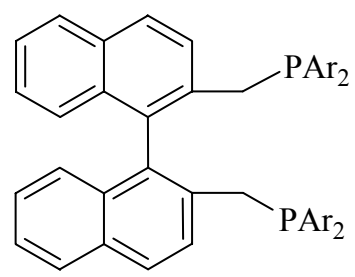

2<smiles>[R]N1C(c2cccc(C3=N[C@@H](c4ccccc4)[C@H](c4ccccc4)N3[R])n2)=N[C@@H](c2ccccc2)[C@@H]1c1ccccc1</smiles>

5<smiles>[Al]C([AlH2])c1nccn1[AlH2]</smiles>

$\mathrm{X}=\mathrm{N} ; \mathrm{CH}_{2}$

3

Scheme 1. A selection of ligands developed in our laboratory.

For more than a decade the development of synthetic methods and the application of catalysis for the preparation of biologically active compounds have attracted our interest (Figure 1). In this respect palladium-catalysis ${ }^{4}$ and amination reactions ${ }^{5}$ have been particularly investigated over the years.
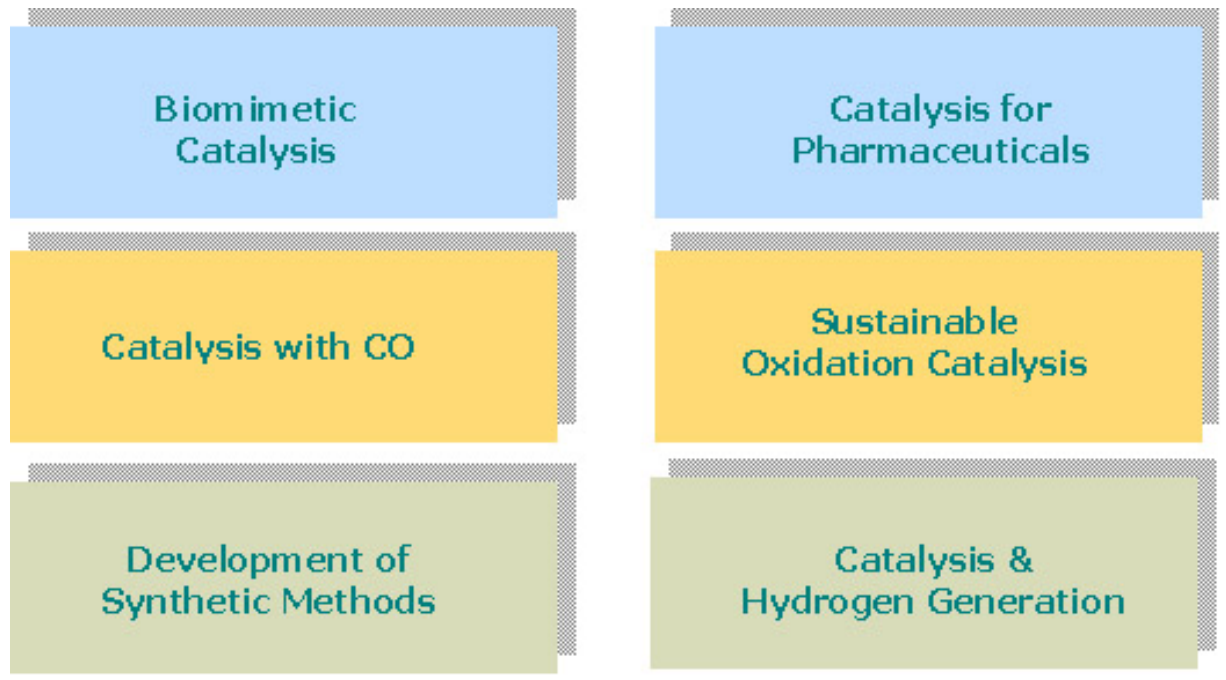

Figure 1. Our research areas of interest. 
Since 1998 catalysis with carbon monoxide ${ }^{6}$ and oxidation catalysis ${ }^{7}$ using molecular oxygen or hydrogen peroxide became prominent areas. Most recently, we initiated a program on the use of homogeneous catalysis for hydrogen generation and biomimetic catalysis, which is based on Fe-catalysis. An important goal of all our work is the facilitation of innovative processes by transfer of basic research to an industrially useful level.

\section{Results and Discussion}

\section{Synthesis of novel binaphthophosphepines}

Clearly, transition metal-catalyzed asymmetric reactions offer efficient and elegant possibilities for the synthesis of enantiomerically pure compounds. ${ }^{8}$ Among the different transition metalcatalyzed asymmetric reactions, hydrogenations of olefins, imines and ketones have been used extensively in the last two decades and are likely to provide the most important access to chiral pharmaceutical intermediates. ${ }^{9}$ In general, optically active phosphine ligands have been viewed as essential in order to achieve high enantioselectivity in asymmetric hydrogenation reactions. ${ }^{8}$ Inspired by the seminal work of Reetz et al., ${ }^{10}$ Feringa and de Vries et al., ${ }^{11}$ as well as Pringle and Claver et al., ${ }^{12}$ we became interested in the synthesis and catalytic application of chiral monodentate phosphines with a 4,5-dihydro-3H-dinaphtho[2,1-c;1',2'-e]phosphepine motif 6 (Scheme 2). ${ }^{13}$ The first example of this class of ligands has been prepared by Gladiali et al. ${ }^{14}$ in the mid 90's. Parallel to the work of Zhang ${ }^{15}$ we established the use of $\mathbf{6}$ as enantioselective ligands for asymmetric hydrogenation of $\alpha$-amino acid precursors, itaconic esters, ${ }^{16}$ enamides, ${ }^{17}$ and $\beta$-ketoesters. ${ }^{18}$ Since our start we have prepared a variety of monodentate ligands, which are prepared in either 1 or 2 steps from optically pure 2,2'-dimethylbinaphthyl (ee $>99 \%$ ). This building block is accessible on a $20 \mathrm{~g}$ scale from 2,2'-binaphthol via esterification with trifluoromethanesulfonic acid anhydride in the presence of pyridine (99\% yield) and subsequent nickel-catalyzed Kumada coupling with methylmagnesium bromide in diethyl ether (95\% yield). 


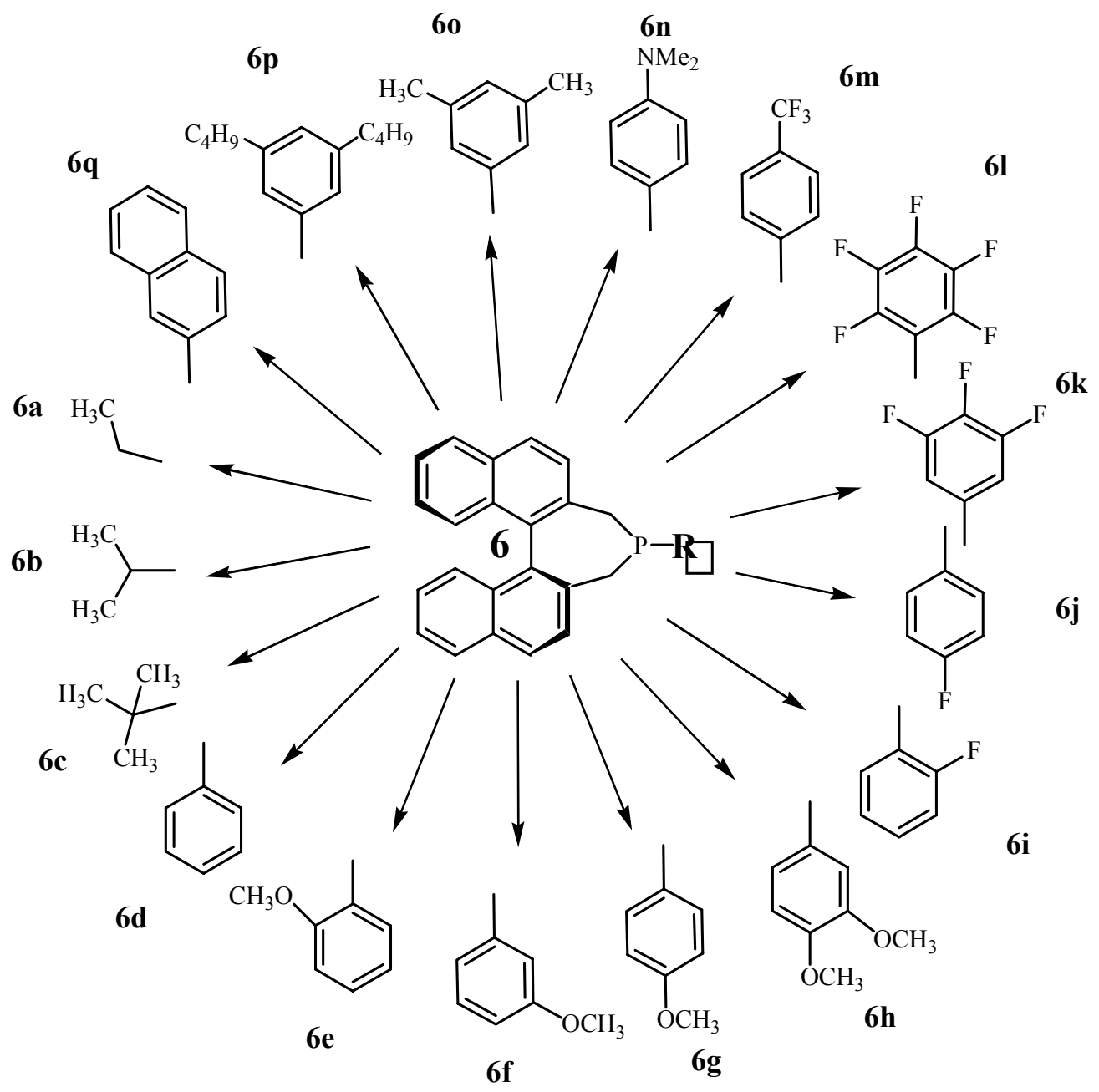

Scheme 2. Ligand library of 4,5-dihydro-3H-dinaphtho[2,1-c;1',2'-e]phosphepines 6.

Typically, our aryl-substituted monodentate ligands gave enantioselectivities of $90-95 \%$ in standard benchmark asymmetric hydrogenations. Recently, in order to improve the enantioselectivity further, we considered the synthesis of bidentate 4,5-dihydro-3Hdinaphtho[2,1-c;1',2'-e]phosphepines. ${ }^{19}$ As building blocks for the novel bidentate ligands we used A, B and 1,1'-binaphthol (Figure 3; ligands 7 and 8) as well as 2,2'-dimethyl-1,1'-binaphthyl (Figure 3; ligands 9 and 10). 

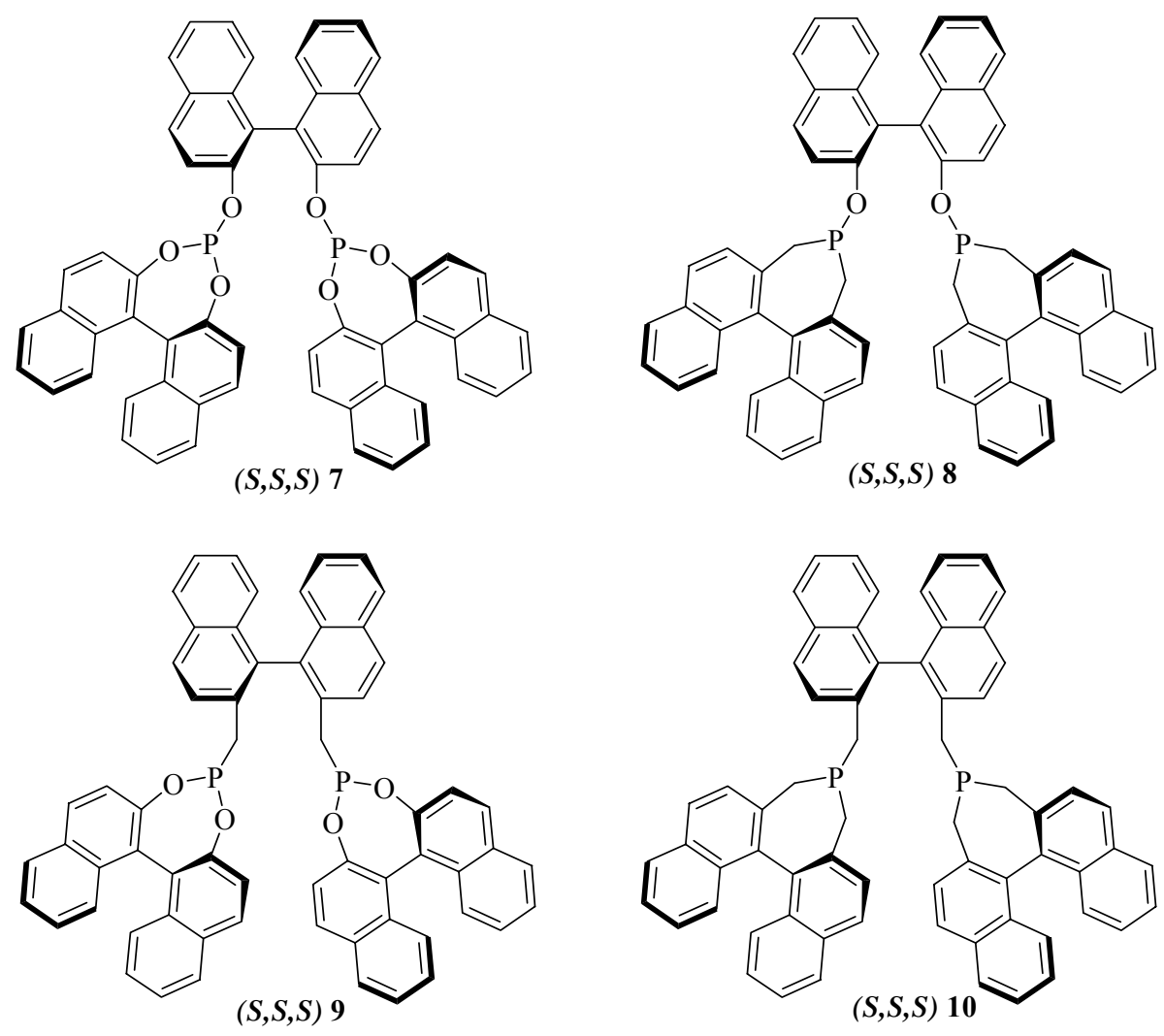

Figure 2. Novel chelating P-ligands.

All ligands with the exception of 9 have been synthesized in the $S, S, S$ - and $S, R, S$ - forms by using the $S$ - and $R$ - configuration of 1,1'-binaphthol and 2,2'-dimethyl-1,1'-binaphthyl bridge to identify the matched and the mismatched combinations (Figure 2).

Ligands $(S, S, S)-7$ and $(S, R, S)-7$ were easily prepared according to literature protocols. ${ }^{20-22}$ The diphosphonites $\mathbf{8}$ were obtained in good yield by reaction of the building block $S$-B with $S$ or $R$-binaphthol in the presence of 1.1 equiv. of triethylamine. The crude product was purified by recrystallization as the rhodium complex $[\mathrm{Rh}(\operatorname{cod}) \mathbf{8}] \mathrm{BF}_{4}$. For the synthesis of the corresponding phosphonites 9 and phosphines 10 the two methyl groups of 2,2'-dimethyl-1,1'-binaphthyl were lithiated selectively to form dark violet crystals. ${ }^{21}$ Subsequently, chlorides A or B are quenched with the dilithium salt to produce the corresponding chelating ligands in high yield. 


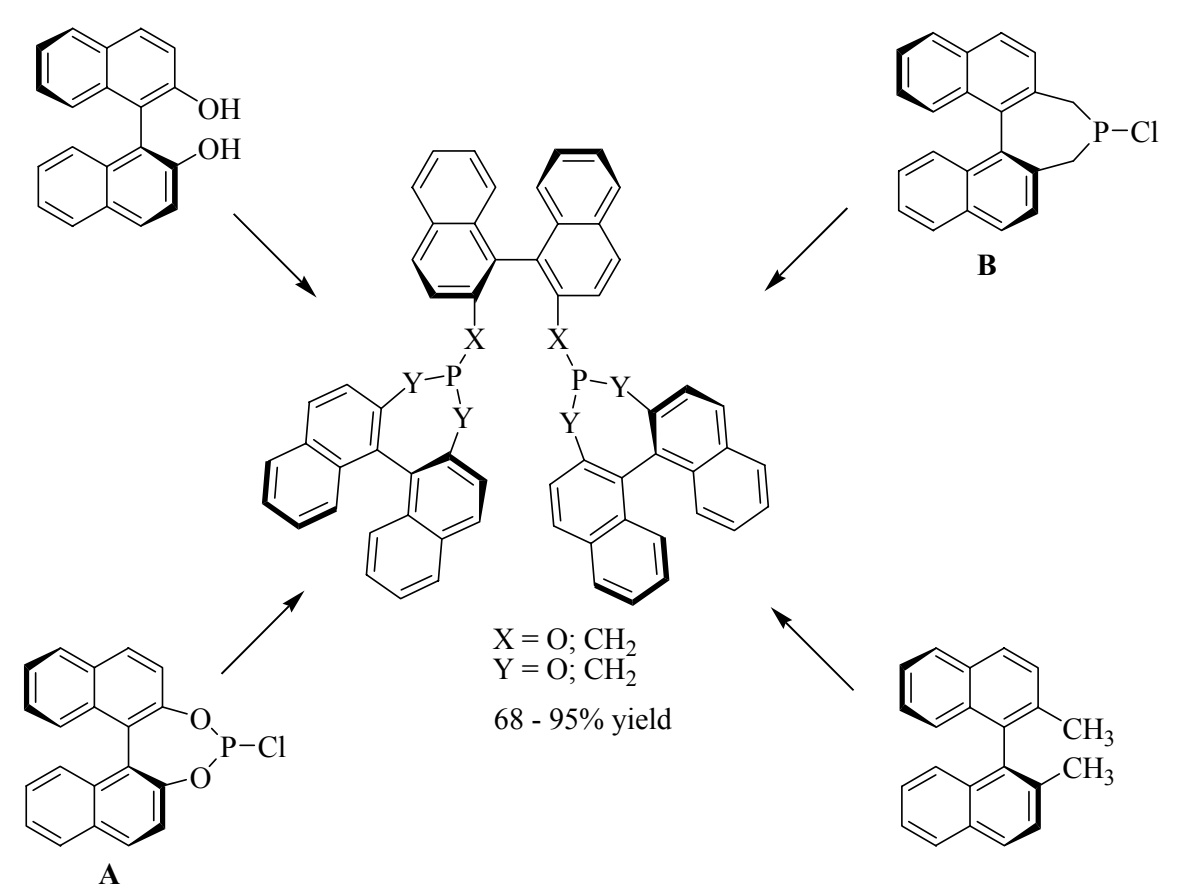

Figure 3. Synthesis of chelating P-ligands.

In summary, we have synthesized a small pool of chelating ligands by replacing oxygen against carbon, step by step, allowing us a systematic investigation of the structural influence on the catalytic behaviour. Prior to our work ligands 8-9 had not been prepared, while $\mathbf{7}$ and $\mathbf{1 0}$ had not been used for asymmetric hydrogenation reactions. The diphosphite $(S, S, S)-7$ was first disclosed by Pringle and co-workers, ${ }^{22}$ who used a Ni(0) complex of 7 for the hydrocyanation of norbornene with acetone cyanohydrine, giving the corresponding product in up to $38 \%$ ee. Another application of $(S, S, S)-7-\mathrm{Cu}(\mathrm{OTf})_{2}$ was reported by Chan et al. ${ }^{20}$ for the conjugate addition of diethylzinc to enones, where enantioselectivities up to $90 \%$ were achieved. In addition, Bakos ${ }^{23}$ and $\mathrm{Chan}^{24}$ tested the diphosphites 7 in the asymmetric hydroformylation of styrene and vinyl acetate, respectively. Buchwald et al. used a cobalt complex in combination with 7 as catalyst for intramolecular asymmetric Pauson-Khand reactions to obtain cyclopentenones in up to $75 \%$ enantiomeric excess. ${ }^{25}$ The conjugate addition of arylboronic acids to dehydroalanine derivatives has been successfully carried out (up to $72 \%$ ee) in the presence of $(R, R, R)-7^{26}$ The synthesis of diphosphine $\mathbf{1 0}$ as a diastereomeric mixture is also known in the literature. ${ }^{27}$ Further bidentate ligands bearing the phosphepine unit $\mathbf{A}$ connected by phenyl or ferrocenyl bridges are known from the elegant work of Zhang et al. as binaphane and f-binaphane. ${ }^{19}$

Our initial catalytic studies focused on the asymmetric hydrogenation of $\alpha$-aminoacid derivatives and itaconic acid dimethyl ester. Typically, catalytic experiments were carried out with $1 \mathrm{~mol} \%$ of the catalyst $\left[\mathrm{Rh}(\mathrm{cod}) \mathrm{L} \mathrm{BF}_{4}\right.$ which was prepared in situ from $0.01 \mathrm{mmol}$ $\left[\mathrm{Rh}(\operatorname{cod})_{2}\right] \mathrm{BF}_{4}$ and 1.1 equiv. of ligands 7-10. Table 1 summarizes the results for the asymmetric hydrogenation of methyl $\alpha$-acetamidocinnamate $\mathbf{1 1}$ in toluene and ethyl acetate. In general, the 
$S, S, S$ - configured ligands produce higher enantioselectivity than the $S, R, S$ - configured ones. This trend dramatically finds its expression for the phosphonites 8 (Table 1, entries 5 and 6) where $(S, S, S)-8$ gave $95 \%$ ee and $(S, R, S)-8$ led to the racemic mixture. Obviously the $S, S, S$-form of the ligand constitutes the matched- and the $S, R, S$-form the mis-matched case. However, both combinations of phosphepine $10(S, S, S$ and $S, R, S)$ hydrogenated methyl $\alpha$-acetamidocinnamate 11 with moderate enantioselectivity (between $38 \%$ and $47 \%$, Table 1, entries 11-14). Regarding the catalyst activity, the $(S, S, S)$-ligands $\mathbf{8}$ and $\mathbf{1 0}$ gave full conversion for this substrate within less than $1 \mathrm{~h}$. On the other hand, especially ligand 7 and 9 hydrogenate so slowly that the reaction was stopped after 24 hours. The best enantioselectivity $(96 \%$ ee) was achieved in ethyl acetate by applying $(S, S, S)-8$ (Table 1 , entry 7 ) which bears P-O bonds in the two sevenmembered rings, and $\mathrm{P}-\mathrm{C}$ bonds in the bridge.

Table 1. Asymmetric hydrogenation of methyl $\alpha$-acetamidocinnamate 11

\begin{tabular}{|c|c|c|c|c|c|c|}
\hline Entry & Ligand & Configuration & Solvent & $t / 2[\mathrm{~min}]$ & Conversion [\%] & $e e[\%](R)$ \\
\hline 1 & 7 & $S, S, S$ & EtOAc & --- & 39 & 27 \\
\hline 2 & 7 & $S, R, S$ & EtOAc & --- & 67 & 18 \\
\hline 3 & 7 & $S, S, S$ & Toluene & --- & 18 & 55 \\
\hline 4 & 7 & $S, R, S$ & Toluene & --- & 39 & 14 \\
\hline 5 & 8 & $S, S, S$ & EtOAc & 5 & $>99$ & 95 \\
\hline 6 & 8 & $S, R, S$ & EtOAc & --- & 16 & Rac. \\
\hline 7 & 8 & $S, S, S$ & Toluene & 540 & $>99$ & 96 \\
\hline 8 & 8 & $S, R, S$ & Toluene & --- & 10 & Rac. \\
\hline 9 & 9 & $S, S, S$ & EtOAc & --- & 79 & 45 \\
\hline 10 & 9 & $S, S, S$ & Toluene & --- & 24 & 26 \\
\hline 11 & 10 & $S, S, S$ & EtOAc & 3 & $>99$ & 38 \\
\hline 12 & 10 & $S, R, S$ & EtOAc & 107 & $>99$ & 43 \\
\hline 13 & 10 & $S, S, S$ & Toluene & 48 & $>99$ & 47 \\
\hline 14 & 10 & $S, R, S$ & Toluene & -- & $>99$ & 40 \\
\hline
\end{tabular}

Reaction conditions: $1.0 \mathrm{mmol}$ substrate; $0.01 \mathrm{mmol}\left[\mathrm{Rh}(\operatorname{cod})_{2}\right] \mathrm{BF}_{4}$; cat. Rh:ligand $=1: 1 ; 15 \mathrm{~mL}$ solvent; $25^{\circ} \mathrm{C}, 1$ bar $\mathrm{H}_{2}$. 
Table 2. Asymmetric hydrogenation of methyl $\alpha$-acetamidoacrylate $\mathbf{1 3}$

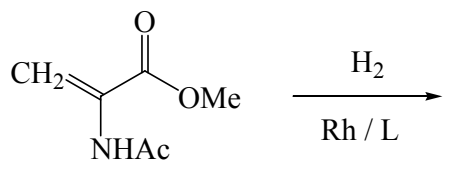

13<smiles>COC(=O)[C@H](C)N</smiles>

14

\begin{tabular}{|c|c|c|c|c|c|c|}
\hline Entry & Ligand & Configuration & Solvent & $t / 2[\mathrm{~min}]$ & Conversion [\%] & $e e[\%](R)$ \\
\hline 1 & 7 & $S, S, S$ & EtOAc & --- & 51 & 6 \\
\hline 2 & 7 & $S, R, S$ & EtOAc & --- & 96 & 17 \\
\hline 3 & 7 & $S, S, S$ & Toluene/SDS & --- & 38 & 42 \\
\hline 4 & 7 & $S, R, S$ & Toluene & --- & 41 & 26 \\
\hline 5 & 8 & $S, S, S$ & EtOAc & 2 & $>99$ & 98 \\
\hline 6 & 8 & $S, R, S$ & EtOAc & --- & 96 & Rac. \\
\hline 7 & 8 & $S, S, S$ & Toluene & 22 & $>99$ & 97 \\
\hline 8 & 8 & $S, R, S$ & Toluene & --- & 40 & Rac. \\
\hline 9 & 9 & $S, S, S$ & EtOAc & --- & $>99$ & 30 \\
\hline 10 & 9 & $S, S, S$ & Toluene & --- & $>99$ & 3 \\
\hline 11 & 10 & $S, S, S$ & THF & 32 & $>99$ & 38 \\
\hline 12 & 10 & $S, R, S$ & THF & 60 & $>99$ & 61 \\
\hline 13 & 10 & $S, S, S$ & EtOAc & 4 & $>99$ & 12 \\
\hline 14 & 10 & $S, R, S$ & EtOAc & --- & $>99$ & 37 \\
\hline 15 & 10 & $S, S, S$ & Toluene & 38 & $>99$ & 23 \\
\hline 16 & 10 & $S, R, S$ & Toluene/SDS & 102 & $>99$ & 69 \\
\hline
\end{tabular}

Reaction conditions: $1.0 \mathrm{mmol}$ substrate; $0.01 \mathrm{mmol}\left[\mathrm{Rh}(\operatorname{cod})_{2}\right] \mathrm{BF}_{4}$; cat. $\mathrm{Rh}$ :ligand $=1: 1 ; 15 \mathrm{~mL}$ solvent; $25^{\circ} \mathrm{C}, 1$ bar $\mathrm{H}_{2}$.

Next, we investigated our ligand pool in the asymmetric hydrogenation of methyl $\alpha-$ acetamidoacrylate 13. Based on previous solvent optimization studies the reaction was carried out in toluene, toluene+SDS (sodium dodecylsulfonate), THF, and ethyl acetate. Selected results for compounds 7-10 are given in Table 2. In agreement with the hydrogenation of methyl $\alpha$ acetamidocinnamate 11 ligand (S,S,S)-8 led to the best result. Enantioselectivities between 97\% and $98 \%$ respectively (Table 2 , entries 5 and 7 ) are obtained, while the isomeric $(S, R, S)-8$ gave only the racemic product! These results clearly show the importance of the right combination of 
the different chiral elements within the ligand. With respect to activity, in most cases complete conversion was observed in reasonable times.

Table 3. Asymmetric hydrogenation of $\alpha$-amino acid derivatives with ligand $(S, S, S)-8$
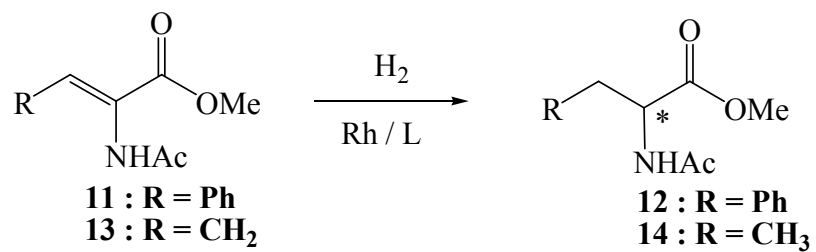

\begin{tabular}{cccccc}
\hline Entry & Substrate & Solvent & $t / 2[\mathrm{~min}]$ & Conversion $[\%]$ & ee $[\%](R)$ \\
\hline 1 & $\mathbf{1 1}$ & EtOAc & 5 & $>99$ & 95 \\
$2^{\#}$ & $\mathbf{1 1}$ & EtOAc & 6 & $>99$ & 94 \\
3 & $\mathbf{1 1}$ & THF & 7 & $>99$ & 90 \\
4 & $\mathbf{1 1}$ & Toluene & 540 & $>99$ & 96 \\
$5^{\#}$ & $\mathbf{1 1}$ & Toluene & 7 & $\mathbf{9 8}$ \\
6 & $\mathbf{1 1}$ & Toluene/SDS & 480 & $>99$ & 92 \\
$7^{\#}$ & $\mathbf{1 1}$ & Toluene/SDS & 28 & $>99$ & $\mathbf{9 8}$ \\
$8^{\#}$ & $\mathbf{1 3}$ & EtOAc & 2 & $>99$ & 96 \\
$9^{\#}$ & $\mathbf{1 3}$ & EtOAc & 3 & $>99$ & 97 \\
10 & $\mathbf{1 3}$ & THF & 3 & $>99$ & 97 \\
11 & $\mathbf{1 3}$ & Toluene & 22 & $>99$ & $\mathbf{9 8}$ \\
$12^{\#}$ & $\mathbf{1 3}$ & Toluene & 2 & $>99$ & 94 \\
13 & $\mathbf{1 3}$ & Toluene/SDS & 32 & $>99$ & 97 \\
$14^{\#}$ & $\mathbf{1 3}$ & Toluene/SDS & 5 & $>99$ & \\
\hline
\end{tabular}

Reaction conditions: $1.0 \mathrm{mmol}$ substrate; $0.01 \mathrm{mmol}\left[\mathrm{Rh}(\operatorname{cod})_{2}\right] \mathrm{BF}_{4}$; cat. Rh:ligand =1:1; $15 \mathrm{~mL}$ solvent; $25^{\circ} \mathrm{C}, 1$ bar $\mathrm{H}_{2}$. ${ }^{\#}$ isolated $[\mathrm{Rh}(\operatorname{cod}) \mathbf{8}] \mathrm{BF}_{4}$.

It is noteworthy that, with the exception of ligands $\mathbf{8}$, the general predefinition of the matched and the mismatched combination of the $R$ - and $S$-configured building blocks is not clear for the hydrogenation of methyl $\alpha$-acetamidoacrylate 13. In the asymmetric hydrogenation of dimethyl itaconate only moderate enantioselectivities were achieved with all ligands. For example $(S, S, S)$ 7 and $(S, S, S)-8$ gave $32 \%$ and $39 \%$ ee, respectively. 
After exploring the catalytic potential of the ligands in the hydrogenation of amino-acid precursors we were interested in the behaviour of $(S, S, S)-\mathbf{8}$ under different reaction conditions. Table 3 shows a comparison of the in situ catalyst and the isolated complex $[\mathrm{Rh}(\operatorname{cod})(S, S, S)-$ $\mathbf{8}] \mathrm{BF}_{4}$. Both $\alpha$-amino acid derivatives were hydrogenated in up to $98 \%$ ee within short time. Using the isolated complex the catalyst activity is improved. Especially for the in situ catalytic system the hydrogenations run slowly in the non-polar solvent toluene (Table 3, entries 4 and 6). Contrary to our previous experience for asymmetric hydrogenation with monodentate ligands ${ }^{16}$ the addition of SDS to toluene has no significant influence.

Finally, the bidentate phosphepine $\mathbf{1 0}$ was also tested in the ruthenium-catalyzed asymmetric hydrogenation of various $\beta$-ketoesters. Other ligands were not included in this study because of the hydrolysis of the P-O bonds under the reaction conditions. All catalytic experiments were performed with $1 \mathrm{~mol} \%$ catalyst formed in situ from $\left[\mathrm{Ru}(\mathrm{cod})\right.$ methylallyl $\left.\mathrm{l}_{2}\right] / \mathrm{HBr}$ and 1 equiv. of ligand 10 in ethanol or methanol. The results obtained for the hydrogenation of different 1,3diketoesters are summarized in Table 4. In general, the $\beta$-hydroxyesters were obtained in good yield, although with low to mediocre enantioselectivity. The best result $(78 \% e e)$ is observed for the phenyl-substituted ketoester (Table 4, entry 8) using the phosphepine 10 in the $S, R, S$ configuration.

Table 4. Asymmetric hydrogenation of $\beta$-ketoesters with ligand 10
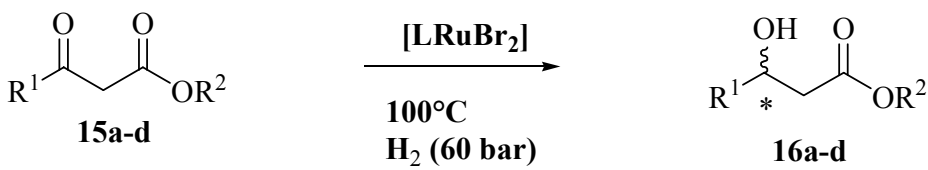

\begin{tabular}{lccccccc}
\hline Entry & Config. of 10 & Substrate & $\mathrm{R}^{1}$ & $\mathrm{R}^{2}$ & $t[\mathrm{~h}]$ & Yield [\%] & ee [\%] \\
\hline 1 & $S, S, S$ & $\mathbf{1 5 a}$ & $\mathrm{CH}_{3}$ & $\mathrm{CH}_{3}$ & 16 & 93 & $27(S)$ \\
2 & $S, S, S$ & $\mathbf{1 5 b}$ & $\mathrm{CH}_{3} \mathrm{CH}_{2}$ & $\mathrm{CH}_{3}$ & 16 & 94 & $34(S)$ \\
3 & $S, S, S$ & $\mathbf{1 5 c}$ & $\mathrm{Cl}^{-\mathrm{CH}_{2}}$ & $\mathrm{CH}_{3} \mathrm{CH}_{2}$ & 16 & 66 & $11(R)$ \\
4 & $S, S, S$ & $\mathbf{1 5 d}$ & $\mathrm{C}_{6} \mathrm{H}_{5}$ & $\mathrm{CH}_{3} \mathrm{CH}_{2}$ & 16 & 99 & $16(R)$ \\
5 & $S, R, S$ & $\mathbf{1 5 a}$ & $\mathrm{CH}_{3}$ & $\mathrm{CH}_{3}$ & 24 & 92 & $52(R)$ \\
6 & $S, R, S$ & $\mathbf{1 5 b}$ & $\mathrm{CH}_{3} \mathrm{CH}_{2}$ & $\mathrm{CH}_{3}$ & 24 & 92 & $43(R)$ \\
7 & $S, R, S$ & $\mathbf{1 5 c}$ & $\mathrm{Cl}_{-} \mathrm{CH}_{2}$ & $\mathrm{CH}_{3} \mathrm{CH}_{2}$ & 24 & 87 & $14(R)$ \\
8 & $S, R, S$ & $\mathbf{1 5 d}$ & $\mathrm{C}_{6} \mathrm{H}_{5}$ & $\mathrm{CH}_{3} \mathrm{CH}_{2}$ & 24 & 99 & $78(S)$ \\
\hline
\end{tabular}

Reaction conditions: methanol or ethanol $(2 \mathrm{~mL}) ; 3.8 \times 10^{-1} \mathrm{mmol} \beta$-ketoester $15 \mathbf{a}-\mathbf{d} ; 3.8 \times 10^{-3}$ mmol Ru(cod)methylallyl ${ }_{2} /$ methanolic $\mathrm{HBr} ; 3.8 \times 10^{-3} \mathrm{mmol} \mathrm{10} ; 60$ bar; $100^{\circ} \mathrm{C}$. 


\section{Conclusions}

In conclusion, we have synthesized a library of novel bidentate chiral ligands containing 4,5dihydro-3H-dinaphtho[2,1-c;1',2'-e]phosphepine and binaphthol building blocks. These ligands can be synthesized easily from 2,2'-binaphthol and 2,2'-dimethylbinaphthol. One of the prepared ligands $((S, S, S)-8)$ can be used efficiently for the rhodium-catalyzed hydrogenation of $\alpha$-amino acid precursors, and enantioselectivities up to $98 \%$ ee were achieved.

\section{Experimental Section}

General Procedures. ${ }^{1} \mathrm{H}-,{ }^{13} \mathrm{C}$ - and ${ }^{31} \mathrm{P}$ - NMR spectra were recorded on a Bruker Spectrometer ARX $400\left({ }^{1} \mathrm{H}, 400.13 \mathrm{MHz} ;{ }^{13} \mathrm{C}, 100.6 \mathrm{MHz} ;{ }^{31} \mathrm{P}, 162.0 \mathrm{MHz}\right)$. The calibration of ${ }^{1} \mathrm{H}-$ and ${ }^{13} \mathrm{C}$ - spectra was carried out on solvent signals $\left(\delta\left(\mathrm{CDCl}_{3}\right)=7.27\right.$ and $77.0 ; \delta\left(\mathrm{C}_{6} \mathrm{D}_{6}\right)=7.15$ and $128.0 ; \delta\left(\mathrm{CD}_{3} \mathrm{COCD}_{3}\right)=2.05$ and 29.8$)$. The ${ }^{31} \mathrm{P}$ - NMR chemical shifts are referenced to $85 \%$ $\mathrm{H}_{3} \mathrm{PO}_{4}$. The ${ }^{1} \mathrm{H}$ - and ${ }^{13} \mathrm{C}$ - NMR signals were assigned by DEPT spectra. Mass spectra were recorded on an AMD 402 spectrometer. Elemental analyses were performed using a Leco CHNS-932. Optical rotations were measured on a Gyromat-HP polarimeter. IR spectra were recorded on $\mathrm{KBr}$ pellets or Nujol mulls on a Nicolet Magna 550. All experiments were carried out under an argon atmosphere. The building blocks $\mathbf{A}$ and $\mathbf{B}$ have been synthesized according to the literature. ${ }^{16,20,21,28}$ Enantiomerically pure 2,2'-dimethylbinaphthyl is obtained in $92 \%$ overall yield from optically pure 2,2'-binaphthol via esterification with trifluoromethanesulfonic acid anhydride in pyridine and subsequent Ni-catalyzed Kumada coupling with $\mathrm{MeMgBr} .{ }^{29}$ Metallation ${ }^{30}$ of enantiomerically pure 2,2'-dimethylbinaphthyl with $n$-BuLi/TMEDA affords the crystalline dilithio species in $70-80 \%$ yield.

\section{General procedure for ligand synthesis}

\section{General procedure for the synthesis of 7 and 8}

To 1 equiv. of 2,2'-binaphthol and 2 equiv. of $\mathrm{NEt}_{3}$ in toluene were added 2 equiv. of building block $\mathbf{A}$ or $\mathbf{B}$ at $0^{\circ} \mathrm{C}$. After stirring the mixture for $2 \mathrm{~h}$ at room temperature and filtration of the precipitate the solvent is evaporated. The crude product is crystallized from ethanol/hexane (1) or THF/hexane (2).

\section{General procedure for the synthesis of 9 and 10}

A suspension of 1 equiv. of the dilithium salt of 2,2'-dimethyl-1,1'-binaphthyl in toluene is added through a tube to 2 equiv. of building block $\mathbf{A}$ or $\mathbf{B}$ dissolved in toluene, over a period of 30-45 $\min$. The color of the reaction mixture turned from dark violet to yellow. After stirring for $10 \mathrm{~h}$, $\mathrm{LiCl}$ was filtered off under inert conditions and washed with toluene. The solvent was evaporated and the crude product purified by flash column chromatography in dry toluene. 
Selected data for $(\boldsymbol{S}, \boldsymbol{S}, \boldsymbol{S})-7$ containing 0.6 mol EtOH. Yield: $82 \%$. ${ }^{1} \mathrm{H}-\mathrm{NMR}(400 \mathrm{MHz}$, $\left.\mathrm{CDCl}_{3}\right): \delta=1.15\left(\mathrm{t}, 1.8 \mathrm{H}, J=7.0 \mathrm{~Hz}, \mathrm{CH}_{3}\right) ; 2.45(\mathrm{~s}, 0.6 \mathrm{H}, \mathrm{OH}) ; 3.63\left(\mathrm{dd}, 1.2 \mathrm{H}, \mathrm{CH}_{2}\right) ; 6.45(\mathrm{~d}$, $2 \mathrm{H}, J=8.8 \mathrm{~Hz}) ; 7.15-7.22(\mathrm{~m}, 14 \mathrm{H}) ; 7.25-7.33(\mathrm{~m}, 8 \mathrm{H}) ; 7.45(\mathrm{~d}, 2 \mathrm{H}, J=8.8 \mathrm{~Hz}) ; 7.65(\mathrm{~d}$, $2 \mathrm{H}, J=8.1 \mathrm{~Hz}) ; 7.73(\mathrm{dd}, 2 \mathrm{H}, J=8.8 \mathrm{~Hz}, J=7.9 \mathrm{~Hz}) ; 7.81(\mathrm{~d}, 2 \mathrm{H}, J=8.1 \mathrm{~Hz}) ; 7.88(\mathrm{~d}, 2 \mathrm{H}, J=$ $8.9 \mathrm{~Hz}) .{ }^{13} \mathrm{C}-\mathrm{NMR}\left(100.6 \mathrm{MHz}, \mathrm{CDCl}_{3}\right): \delta=121.1 ; 121.7 ; 121.8 ; 122.4 ; 124.3 ; 124.6 ; 124.9$; $125.1 ; 125.8 ; 126.1 ; 126.2 ; 126.8 ; 126.9 ; 128.0 ; 128.2 ; 128.3 ; 129.5 ; 130.0 ; 130.2 ; 130.8 ; 131.0 ;$ $131.4 ; 132.2 ; 132.6 ; 134.2 ; 146.9 ; 147.5 ; 148.5 .{ }^{31} \mathrm{P}-\mathrm{NMR}\left(162 \mathrm{MHz}, \mathrm{CDCl}_{3}\right): \delta=145.2$. IR (KBr): 3423w; 3055m;1619m;1589m;1505m;1463m;1431m;1359m;1325m;1217s; 1189m; 1154m; 1069m; 978m; 943s; 865m; 825s; 785s; 770m; 751s; 696m; 682m; 644w; 629w; 599w; $569 \mathrm{w} ; 556 \mathrm{~m} ; 526 \mathrm{~m}$. MS (FAB): $\mathrm{m} / \mathrm{z}(\%)=916\left(\left[\mathrm{M}^{+}+2\right], 45\right) ; 664(5) ; 601(13) ; 583(82) ; 315$ (95). Mp $188^{\circ} \mathrm{C}$. Optical Rotation: $[\alpha]^{23}=+337(\mathrm{c}=0.5$; toluene).

Selected data for $(\boldsymbol{S}, \boldsymbol{R}, \boldsymbol{S})-7$ containing $0.8 \mathrm{~mol}$ EtOH. Yield 95\%. ${ }^{1} \mathrm{H}-\mathrm{NMR}(400 \mathrm{MHz}$, $\left.\mathrm{CDCl}_{3}\right): \delta=1.25\left(\mathrm{t}, 2.4 \mathrm{H}, J=7.0 \mathrm{~Hz}, \mathrm{CH}_{3}\right) ; 2.65(\mathrm{~s}, 0.8 \mathrm{H}, \mathrm{OH}) ; 3.75\left(\mathrm{dd}, 2.4 \mathrm{H}, \mathrm{CH}_{2}\right) ; 5.8(\mathrm{~d}$, $2 \mathrm{H}, J=8.7 \mathrm{~Hz}) ; 7.2-7.3(\mathrm{~m}, 14 \mathrm{H}) ; 7.35-7.43(\mathrm{~m}, 8 \mathrm{H}) ; 7.6(\mathrm{~d}, 2 \mathrm{H}, J=8.7 \mathrm{~Hz}) ; 7.75(\mathrm{~d}, 2 \mathrm{H}, J$ $=7.1 \mathrm{~Hz}) ; 7.87(\mathrm{~d}, 4 \mathrm{H}, J=8.7 \mathrm{~Hz}) ; 8.05(\mathrm{~d}, 2 \mathrm{H}, J=8.3 \mathrm{~Hz}) ; 8.1(\mathrm{~d}, 2 \mathrm{H}, J=8.9 \mathrm{~Hz}) .{ }^{13} \mathrm{C}-\mathrm{NMR}$ $\left(100.6 \mathrm{MHz}, \mathrm{CDCl}_{3}\right): \delta=121.1 ; 121.4 ; 121.7 ; 122.4 ; 123.0 ; 124.0 ; 124.3 ; 124.6 ; 125.0 ; 125.2 ;$ $125.8 ; 126.1 ; 126.3 ; 126.8 ; 126.9 ; 128.0 ; 128.1 ; 128.2 ; 129.1 ; 130.1 ; 130.3 ; 131.0 ; 131.1 ; 131.4$; $132.1 ; 132.7 ; 134.0 ; 147.0 ; 147.3 ; 148.2 .{ }^{31} \mathrm{P}-\mathrm{NMR}\left(162 \mathrm{MHz}, \mathrm{CDCl}_{3}\right): \delta=145.8 . \mathrm{IR}(\mathrm{KBr})$ : $3448 \mathrm{~m} ; 3055 \mathrm{~m} ; 1620 \mathrm{~m} ; 1589 \mathrm{~m} ; 1507 \mathrm{~m} ; 1463 \mathrm{~m} ; 1431 \mathrm{~m} ; 1359 \mathrm{~m} ; 1326 \mathrm{~m} ; 1215 \mathrm{~s} ; 1189 \mathrm{~m}$; $1154 \mathrm{~m} ; 1070 \mathrm{~m} ; 979 \mathrm{~m} ; 950 \mathrm{~s} ; 865 \mathrm{~m} ; 825 \mathrm{~s} ; 787 \mathrm{~s} ; 770 \mathrm{~m} ; 748 \mathrm{~s} ; 694 \mathrm{~m} ; 645 \mathrm{w} ; 630 \mathrm{w} ; 598 \mathrm{w} ; 569 \mathrm{w}$; 556w; 526m. Mp $189^{\circ} \mathrm{C}$. Optical Rotation: $[\alpha]^{23}=+194(\mathrm{c}=0.5$; toluene).

Selected data for $(\boldsymbol{S}, \boldsymbol{S}, \boldsymbol{S})-8$. Yield: $60 \% .{ }^{1} \mathrm{H}-\mathrm{NMR}\left(400 \mathrm{MHz}, \mathrm{CDCl}_{3}\right): \delta=1.9-2.09(\mathrm{~m}, 4 \mathrm{H}$, $\left.\mathrm{CH}_{2}\right) ; 2.2-2.3\left(\mathrm{dd}, 3 \mathrm{H}, \mathrm{CH}_{2}\right) ; 2.35\left(\mathrm{~d}, 1 \mathrm{H}, \mathrm{CH}_{2}\right) .{ }^{31} \mathrm{P}-\mathrm{NMR}\left(162 \mathrm{MHz}, \mathrm{CDCl}_{3}\right): \delta=158.0$. IR (KBr): 3423m; 3053m;1619m;1592m;1507s; 1459m;1431m;1343m;1256m;1225s;1144m; $1127 \mathrm{~m} ; 1071 \mathrm{w} ; 1027 \mathrm{w} ; 982 \mathrm{~m} ; 933 \mathrm{~m} ; 865 \mathrm{~m} ; 816 \mathrm{~s} ; 748 \mathrm{~s} ; 694 \mathrm{w} ; 673 \mathrm{w} ; 621 \mathrm{w} ; 566 \mathrm{w} ; 540 \mathrm{w} ;$ 520w. MS (FAB): $m / z(\%)=907\left(\left[\mathrm{M}^{+}\right], 20\right) ; 859(5) ; 779(8) ; 720(10) ; 597(15) ; 495(8) ; 432$ $(10) ; 367(12) ; 310(25) ; 282(40) ; 265(100) ; 198(18)$. Mp $150^{\circ} \mathrm{C}$. Optical rotation: $[\alpha]^{23}=-58$ (c $=0.5$; toluene).

Selected data for $(\boldsymbol{S}, \boldsymbol{R}, \boldsymbol{S})-8$. Yield: $81 \%$. ${ }^{1} \mathrm{H}-\mathrm{NMR}\left(400 \mathrm{MHz}, \mathrm{CDCl}_{3}\right): \delta=2.16-2.25(\mathrm{~m}, 2 \mathrm{H}$, $\left.\mathrm{CH}_{2}\right) ; 2.39-2.51$ (dd, 4H, $\left.\mathrm{CH}_{2}, J=13.95 \mathrm{~Hz}\right) ; 2.89-3.07\left(\mathrm{~m}, 2 \mathrm{H}, \mathrm{CH}_{2}\right) .{ }^{31} \mathrm{P}-\mathrm{NMR}(162 \mathrm{MHz}$, $\left.\mathrm{CDCl}_{3}\right): \delta=156.5$. IR (KBr): 3423s; 3053m;2958m;1619m;1592m;1507s; 1459m;1426m; $1355 \mathrm{~m} ; 1223 \mathrm{~s} ; 1145 \mathrm{~m} ; 1071 \mathrm{w} ; 1027 \mathrm{w} ; 984 \mathrm{~m} ; 933 \mathrm{w} ; 865 \mathrm{~m} ; 814 \mathrm{~s} ; 747 \mathrm{~s} ; 663 \mathrm{w} ; 622 \mathrm{w} ; 567 \mathrm{w} ;$ 520w. MS (FAB): m/z (\%) = $907\left(\left[\mathrm{M}^{+}\right], 20\right) ; 859$ (5); 779 (8); 720 (10); 597 (15); 495 (8); 432 (13); 367 (12); $310(25) ; 282$ (40); 265 (100); 198 (18). Mp $162^{\circ} \mathrm{C}$. Optical rotation: $[\alpha]^{23}=-106$ (c=0.5; toluene).

Selected data for $(\boldsymbol{S}, \boldsymbol{S}, \boldsymbol{S})-9$. Yield: $79 \% .{ }^{1} \mathrm{H}-\mathrm{NMR}\left(400 \mathrm{MHz}, \mathrm{CDCl}_{3}\right): \delta=2.27(\mathrm{~d}, 2 \mathrm{H}, J=13.5$ $\left.\mathrm{Hz}, \mathrm{CH}_{2}\right) ; 2.59$ (t, 2H, $\left.J=14.3 \mathrm{~Hz}, \mathrm{CH}_{2}\right) ; 6.39$ (d, 2H, J=8.8 Hz); $6.39-6.42$ (d, 2H); 6.67 (d, $2 \mathrm{H}, J=8.5 \mathrm{~Hz}) ; 6.74(\mathrm{~d}, 2 \mathrm{H}, J=8.5 \mathrm{~Hz}) ; 6.86(\mathrm{~d}, 2 \mathrm{H}, J=8.5 \mathrm{~Hz}) ; 7.03-7.14(\mathrm{~m}, 8 \mathrm{H}) ; 7.21(\mathrm{~d}$, $2 \mathrm{H}, J=8.7 \mathrm{~Hz}) ; 7.25(\mathrm{~d}, 2 \mathrm{H}, J=8.7 \mathrm{~Hz}) ; 7.31(\mathrm{~m}, 2 \mathrm{H}) ; 7.34(\mathrm{~d}, 2 \mathrm{H}, J=8.0 \mathrm{~Hz}) ; 7.55(\mathrm{~d}, 2 \mathrm{H}, J=$ $8.5 \mathrm{~Hz}) ; 7.71(\mathrm{~d}, 2 \mathrm{H}, J=7,9 \mathrm{~Hz}) ; 7.80(\mathrm{dd}, 2 \mathrm{H}, J=8.1 \mathrm{~Hz}, J=8.7 \mathrm{~Hz}) .{ }^{13} \mathrm{C}-\mathrm{NMR}(100.6 \mathrm{MHz}$, 
$\left.\mathrm{CDCl}_{3}\right): \delta=40.1\left(\mathrm{~d}, \mathrm{CH}_{2}, J=40.0 \mathrm{~Hz}\right) ; 121.5 ; 121.6 ; 123.2 ; 123.9 ; 124.2 ; 124.7 ; 124.8 ; 124.9$; $125.3 ; 125.4 ; 126.1 ; 126.2 ; 126.4 ; 126.6 ; 126.8 ; 127.7 ; 128.0 ; 128.2 ; 129.0 ; 129.9 ; 130.2 ; 130.8$; $131.3 ; 131.6 ; 132.2 ; 132.5 ; 132.8 ; 135.0 ; 147.0 ; 149.0 .{ }^{31} \mathrm{P}-\mathrm{NMR}\left(162 \mathrm{MHz}, \mathrm{CDCl}_{3}\right): \delta=205.8$. IR (KBr): 3517w; 3054m; 2923m; 2857w; 1619s; 1590s; 1507s; 1463s; 1431m; 1359m; 1326m; $1270 \mathrm{~m} ; 1257 \mathrm{~m} ; 1229 \mathrm{~s} ; 1155 \mathrm{~m} ; 1143 \mathrm{~m} ; 1071 \mathrm{~s} ; 1028 \mathrm{w} ; 981 \mathrm{~m} ; 950 \mathrm{~s} ; 865 \mathrm{~m} ; 821 \mathrm{~s} ; 748 \mathrm{~s} ; 730 \mathrm{~m} ;$ 695m; 643w; 629w; 566w; 555m; 527w. MS (EI): m/z (\%) = $596\left(\left[\mathrm{M}^{+}-314\right], 65\right) ; 268(14) ; 181$ (28); 131 (79); 69 (30). Mp $195^{\circ} \mathrm{C}$. Optical Rotation: $[\alpha]^{23}=694$ (c=0.5; toluene).

Selected data for $(\boldsymbol{S}, \boldsymbol{S}, \boldsymbol{S})-\mathbf{1 0}$. Yield: $68 \%$. Calc. for $\mathrm{C}_{66} \mathrm{H}_{48} \mathrm{P}_{2}: \mathrm{C}, 87.66 ; \mathrm{H}, 5.31 ; \mathrm{P}, 6.86 \%$. Found: C, 87.53; H, 5.11; P, 6.43\%. ${ }^{1} \mathrm{H}-\mathrm{NMR}\left(400 \mathrm{MHz}, \mathrm{CDCl}_{3}\right): \delta=2.0-2.6\left(\mathrm{~m}, 12 \mathrm{H}, \mathrm{CH}_{2}\right)$; $6.0-8.0(\mathrm{~m}, 36 \mathrm{H}) .{ }^{13} \mathrm{C}-\mathrm{NMR}\left(100.6 \mathrm{MHz}, \mathrm{CDCl}_{3}\right): \delta=29.7\left(\mathrm{~d}, \mathrm{CH}_{2}\right) ; 31.1\left(\mathrm{~d}, \mathrm{CH}_{2}\right) ; 35.0(\mathrm{~d}$, $\left.\mathrm{CH}_{2}\right) .{ }^{31} \mathrm{P}-\mathrm{NMR}\left(162 \mathrm{MHz}, \mathrm{CDCl}_{3}\right): \delta=9.4$. IR (KBr): 3432w; 3049m; 3007m; 2908m; 1710w; $1618 \mathrm{~m} ; 1592 \mathrm{~m} ; 1507 \mathrm{~s} ; 1421 \mathrm{~m} ; 1358 \mathrm{~m} ; 1327 \mathrm{w} ; 1248 \mathrm{~m} ; 1222 \mathrm{~m} ; 1160 \mathrm{~m} ; 1144 \mathrm{~m} ; 1026 \mathrm{~m} ; 960 \mathrm{~m} ;$ 934m; 865m; 837s; 813s; 747s; 673m; 621m; 566w; 520w. MS (FAB): m/z (\%) = $902\left(\left[\mathrm{M}^{+}-1\right]\right.$, $<5)$; 591 (10); 501 (3); 335 (7); 265 (65); 199 (100). Mp 233 ${ }^{\circ}$ C. Optical Rotation: $[\alpha]^{23}=10(\mathrm{c}=$ 0.5 ; toluene).

Selected data for $(\boldsymbol{S}, \boldsymbol{R}, \boldsymbol{S})-\mathbf{1 0}$. Yield: $70 \%$. Calc. for $\mathrm{C}_{66} \mathrm{H}_{48} \mathrm{P}_{2}$ : C, 87.66; H, 5.31; P, 6.86\%. Found: C, 87.18; H, 5.42; P, 6.34\%. ${ }^{1} \mathrm{H}-\mathrm{NMR}\left(400 \mathrm{MHz}, \mathrm{CDCl}_{3}\right): \delta=2.0-2.7\left(\mathrm{~m}, 12 \mathrm{H}, \mathrm{CH}_{2}\right)$; $6.4-7.9(\mathrm{~m}, 36 \mathrm{H}) .{ }^{31} \mathrm{P}-\mathrm{NMR}\left(162 \mathrm{MHz}, \mathrm{CDCl}_{3}\right): \delta=7.4$. IR $(\mathrm{KBr}): 3432 \mathrm{~s} ; 3052 \mathrm{~m} ; 2958 \mathrm{~m}$; $1618 \mathrm{~m} ; 1594 \mathrm{~m} ; 1508 \mathrm{~s} ; 1406 \mathrm{~m} ; 1359 \mathrm{~m} ; 1329 \mathrm{w} ; 1249 \mathrm{~m} ; 1228 \mathrm{~m} ; 1160 \mathrm{~m} ; 1027 \mathrm{~m} ; 961 \mathrm{w} ; 931 \mathrm{w} ;$ 866m; 816s; 748s; 680w; 622w; 567w; 544w; 520w. MS (FAB): m/z (\%) = $903\left(\left[\mathrm{M}^{+}\right], 15\right) ; 874$ (3); 827 (1); 695 (2); 655 (3); 619 (10); 592 (100); 529 (5); 413 (7). Mp 228 C. Optical Rotation: $[\alpha]^{23}=100(\mathrm{c}=0.33$; toluene $)$.

Preparation of the $\mathbf{R h}(\mathbf{I I})$ complex of $(\boldsymbol{S}, \boldsymbol{S}, \boldsymbol{S})-\mathbf{8}$. A solution of ligand $(S, S, S)-2$ (1.2 mmol) in THF $(7 \mathrm{~mL})$ was added dropwise to a stirred solution of $\mathrm{Rh}(\mathrm{cod}) \mathrm{acac}(1.2 \mathrm{mmol})$ in the same solvent $(5 \mathrm{~mL})$. After $1 \mathrm{~h}$ at $20^{\circ} \mathrm{C}$, ethereal $\mathrm{HBF}_{4}(1.2 \mathrm{mmol})$ is added and the solution stirred for an additional hour. The solvent was removed and the residue crystallized in acetone/methanol to give red-brown solid. Calc. for $\mathrm{C}_{72} \mathrm{H}_{56} \mathrm{O}_{2} \mathrm{P}_{2} \mathrm{RhBF}_{4}$ : C, 71.71; H, 4.65\%. Found: $\mathrm{C}, 71.67 ; \mathrm{H}$, 4.89\%. ${ }^{31} \mathrm{P}-\mathrm{NMR}\left(162 \mathrm{MHz}, \mathrm{CDCl}_{3}\right): \delta=167.5$ (d, $\left.J=173.4 \mathrm{~Hz}\right) . \mathrm{MS}(\mathrm{FAB}): \mathrm{m} / \mathrm{z}(\%)=1117$ $\left(\left[\mathrm{M}^{+}-1-\mathrm{BF}_{4}\right], 60\right) ; 1009\left(\left[\mathrm{M}^{+}-1-\mathrm{BF}_{4}-\mathrm{cod}\right], 70\right) ; 834$ (100); 709 (100).

\section{Catalytic experiments}

Asymmetric hydrogenation of $\alpha$-aminoacids 11 and 13. Hydrogenation was performed under normal pressure at $25^{\circ} \mathrm{C}$. A mixture of $0.01 \mathrm{mmol}\left[\mathrm{Rh}(\operatorname{cod})_{2}\right] \mathrm{BF}_{4}, 0.01 \mathrm{mmol}$ ligand $\mathbf{7 - 1 0}$ and 0.1 mmol methyl $\alpha$-acetamidocinnamate 5 or methyl $\alpha$-acetamidoacrylate 7 was stirred for 15 min in $15 \mathrm{~mL}$ solvent. The hydrogenation flask was flushed several times with hydrogen. The reaction was followed by a volumetric measurement at $25^{\circ} \mathrm{C} \pm 0.5^{\circ} \mathrm{C}$. The enantiomeric excess of 12 (Lipodex E, $150^{\circ} \mathrm{C}$ isotherm) and 14 (Chirasil Val, $120^{\circ} \mathrm{C}$ isotherm) were determined by GC.

Asymmetric hydrogenation of $\boldsymbol{\beta}$-ketoesters 15a-d. Catalytic hydrogenation experiments were carried out in a parallel autoclave $(8 \times 3 \mathrm{~mL})$. In a typical experiment, the autoclave was charged 
with a mixture of $3.8 \times 10^{-3} \mathrm{mmol}$ of catalyst $\left[\mathrm{LRuBr}_{2}\right]$ prepared in situ and $3.8 \times 10^{-1} \mathrm{mmol} \beta$ ketoester 15a-d in $2 \mathrm{~mL}$ methanol or ethanol under an argon stream. The autoclave's contents were stirred at 60 bar hydrogen pressure and $100^{\circ} \mathrm{C}$ for $16-24 \mathrm{~h}$. The autoclave was cooled to room temperature and the hydrogen was released. The reaction mixture was filtered over silica gel and the enantiomeric excess determined by GC (Lipodex E) or HPLC (Chiracel OD-H). Most of the hydrogenation products have been previously described. Methyl 3-hydroxybutyrate (16a): GC $\left(25 \mathrm{~m}\right.$ Lipodex E) $95^{\circ} \mathrm{C}$ isothermal: $t_{\mathrm{r}}(\mathrm{min})=4.9(S), 5.7(R)$; methyl 3-hydroxyvalerate (16b): GC (25 m Lipodex E) $85^{\circ} \mathrm{C}$ isothermal: $t_{\mathrm{r}}(\mathrm{min})=10.9(S), 11.6(R)$; ethyl 3-hydroxy-4chlorobutyrate (16c): GC (25 m Lipodex E) $95^{\circ} \mathrm{C}$ isothermal: $t_{\mathrm{r}}(\mathrm{min})=20.4(R), 20.6(S)$; Ethyl 3-hydroxy-3-phenylpropionate (16d): HPLC (Chiracel OD-H, hexane/ethanol $=$ 95:5, 0.5 $\mathrm{mL} / \mathrm{min}), t_{\mathrm{r}}(\mathrm{min})=10.1(S), 11.5(R)$.

\section{Acknowledgements}

This work has been financed by the State of Mecklenburg-Pomerania and the Bundesministerium für Bildung und Forschung (BMBF). We thank Mrs. C. Voss, Mrs. C. Mewes, Mrs. M. Heyken, Mrs. S. Buchholz and Dr. C. Fischer (all Leibniz-Institut für Katalyse e.V. an der Universität Rostock) for their excellent technical and analytical support.

\section{References and Notes}

1. Selected examples: (a) Zapf, A.; Ehrentraut, A.; Beller, M. Angew. Chem. 2000, 112, 4315; Angew. Chem. Int. Ed. 2000, 39, 4153. (b) Zapf, A.; Jackstell, R.; Rataboul, F.; Riermeier, T.; Monsees, A.; Fuhrmann, C.; Shaik, N.; Dingerdissen, U.; Beller, M. Chem. Commun. 2004, 38. (c) Rataboul, F.; Zapf, A.; Jackstell, R.; Riermeier, T.; Monsees, A.; Dingerdissen, U.; Beller, M. Chem. Eur. J. 2004, 10, 2983. (d) Harkal, S.; Rataboul, F.; Zapf, A.; Fuhrmann, C.; Riermeier, T.; Monsees, A.; Beller, M. Adv. Synth. Catal. 2004, 346, 1742.

2. Bhor, S.; Anilkumar, G.; Tse, M. K.; Döbler, C.; Klawonn, M.; Bitterlich, B.; Grotevendt, A.; Beller, M. Org. Lett. 2005, 7, 3393.

3. Selected examples: (a) Robert H. Grubbs, "Handbook of Metathesis",WileyVCH:Verlag, 2003. (b) Trnka, T. M.; Dias, E. L.; Day, M. W.; Grubbs, R. H. ARKIVOC 2002, (xiii), 28.

4. For a personal account see: Zapf, A.; Beller, M. Chem. Commun. 2005, 431.

5. Reviews: (a) Seayad, J.; Tillack, A.; Hartung, C. G.; Beller, M. Adv. Synth. Catal. 2002, 344, 795. (b) Beller, M.; Breindl, C.; Eichberger, M.; Hartung, C. G.; Seayad, J.; Thiel, O.; Tillack, A.; Trauthwein, H. Synlett 2002, 1579. 
6. Selected examples: (a) Seayad, A.; Ahmed, M.; Klein, H.; Jackstell, R.; Gross, T.; Beller, M. Science 2002, 297, 1676. (b) Moballigh, A.; Seayad, A.; Jackstell, R.; Beller, M. J. Am. Chem. Soc. 2003, 125, 10311. (c) Klein, H.; Jackstell, R.; Beller, M. Chem. Commun. 2005, 2283.

7. (a) Döbler, C.; Mehltretter, G.; Sundermeier, U.; Beller, M. J. Am. Chem. Soc. 2000, 122, 10289. (b) Tse, M. K.; Döbler, C.; Bhor, S.; Klawonn, M.; Mägerlein, W.; Hugl, H.; Beller, M. Angew. Chem. 2004, 116, 5367; Angew. Chem. Int. Ed. 2004, 43, 5255. (c) Tse, M. K.; Klawonn, M.; Bhor, S.; Döbler, C.; Anilkumar, G.; Beller, M. Org. Lett. 2005, 7, 987.

8. Noyori, R. Asymmetric Catalysis in Organic Synthesis, Wiley, New York, 1994. (b) Beller, M.; Bolm, C. (Eds.), Transition Metals for Organic Synthesis, Wiley-VCH: Weinheim, 1998. (c) Jacobsen, E. N.; Pfaltz, A.; Yamamoto, H. (Eds.), Comprehensive Asymmetric Catalysis, Springer:Berlin, 1999.

9. Reviews: (a) MacCarthy, M.; Guiry, P. J. Tetrahedron 2001, 57, 3809. (b) Zhang, X. Enantiomer 1999, 4, 541. (c) Gladiali, S., Fabbri, D. Chem. Ber. Recueil. 1997, 130, 543. (d) Lagasse, F.; Kagan, H. B. Chem. Pharm. Bull. 2000, 48, 315.

10. (a) Reetz, M. T.; Sell, T. Tetrahedron Lett. 2000, 41, 6333. (b) Reetz, M. T.; Mehler, G. Angew. Chem. Int. Ed. 2000, 39, 3889. (c) Reetz, M. T.; Mehler, G.; Meiswinkel, G.; Sell, T. Tetrahedron Lett. 2002, 43, 7941. (d) Reetz, M. T.; Mehler, G.; Meiswinkel, G.; Sell, T. Angew. Chem. Int. Ed. 2003, 42, 787. (e) Reetz, M. T.; Mehler, G. Tetrahedron Lett. 2003, 44, 4593. (f) Reetz, M. T.; Li, X. G. Tetrahedron 2004, 60, 9709. (g) Reetz, M. T.; Mehler, G.; Meiswinkel, A. Tetrahedron: Asymmetry 2004, 15, 2165. (h) Reetz, M. T.; Ma, J.-A.; Goddard, R. Angew. Chem. Int. Ed. 2005, 44, 412.

11. (a) Arnold, L. A.; Imobos, R.; Manoli, A.; de Vries, A. H. M.; Naasz, R.; Feringa, B. Tetrahedron 2000, 56, 2865. (b) van den Berg, M.; Minnaard, A. J.; Schudde, E. P.; van Esch, J.; de Vries, A. H. M.; de Vries, J. G.; Feringa, B. L. J. Am. Chem. Soc. 2000, 122, 11539. (c) Minnaard, A. J.; van den Berg, M.; Schudde, E. P.; van Esch, J.; de Vries, A. H. M.; de Vries, J. G. ; Feringa, B. Chim. Oggi 2001, 19, 12. (d) Pena, D.; Minnaard, A. J.; de Vries, J. G.; Feringa, B. L. J. Am. Chem. Soc. 2002, 124, 14552. (e) van den Berg, M.; Haak, R. M.; Minnaard, A. J.; de Vries, A. H. M.; de Vries, J. G.; Feringa, B. L. Adv. Synth. Catal. 2002, 344, 1003. (f) van den Berg, M.; Minnaard, A. J.; Haak, R. M.; Leeman, M.; Schudde, E. P.; Meetsma, A.; Feringa, B. L.; de Vries, A. H. M.; Maljaars, C. E. P.; Willans, C. E.; Hyett, D.; Boogers, J. A. F.; Henderickx, H. J. W.; de Vries, J. G. Adv. Synth. Catal. 2003, 345, 308. (g) Pena, D.; Minnaard, A. J.; de Vries, A. H. M.; de Vries, J. G.; Feringa, B. L. Org. Lett. 2003, 5, 475. (h) Jiang, X.; van den Berg, M.; Minnaard, A. J.; Feringa, B. L.; de Vries, J. G. Tetrahedron: Asymmetry 2004, 15, 2223.

(i) Eelkema, R.; van Delden, R. A.; Feringa; B. L. Angew. Chem. Int. Ed. 2004, 43, 5013.

(j) Duursma, A.; Boiteau, J.-G.; Lefort, L.; Boogers, J. A. F.; de Vries, A. H. M.; de Vries, J. G.; Minnaard, A. J.; Feringa, B. L. J. Org. Chem. 2004, 69, 8045. (k) 
Bernsmann, H.; van den Berg, M.; Hoen, R.; Minnaard, A. J.; Mehler, G.; Reetz, M. T.; de Vries, J. G.; Feringa, B. L. J. Org. Chem. 2005, 70, 943.

12. (a) Claver, C.; Fernandez, E.; Gillon, A.; Heslop, K.; Hyett, D. J.; Martorell, A.; Orpen, A. G.; Pringle, P. G. Chem. Comm. 2000, 961. (b) Martorell, A.; Naasz, R.; Feringa, B. L.; Pringle, P. G. Tetrahedron: Asymmetry 2001, 12, 2497.

13. Reviews about chiral monodentate phosphorus ligands: (a) Komarov, I. V.; Börner, A. Angew. Chem. Int. Ed. 2001, 40, 1197. (b) Tang, W.; Zhang, X. Chem. Rev. 2003, 103, 3029. (c) Jerphagnon, T.; Renaud, J.-L.; Bruneau, C. Tetrahedron: Asymmetry 2004, 15, 2101. Selected other chiral monodentate ligands: (d) Chen, W.; Xiao, J. Tetrahedron Lett. 2001, 42, 8737. (e) Hu, A.-G.; Fu, Y.; Xie, J.-H.; Zhou, H.; Wang, L.-X.; Zhou, Q.-L. Angew. Chem. Int. Ed. 2002, 41, 2348. (f) Fu, Y.; Xie, J.-H.; Hu, A.-G.; Zhou, H.; Wang, L.-X.; Zhou, Q.-L. Chem. Comm. 2002, 480. (g) Ostermeier, M.; Prieß, J.; Helmchen, G. Angew. Chem. Int. Ed. 2002, 41, 612. (h) Hua, Z.; Vassar, V. C.; Ojima, I. Org. Lett. 2003, 21, 3831. (i) Kinoshita, N.; Marx, K. H.; Tanaka, K.; Tsubaki, K.; Kawabata, T.; Yoshikai, N.; Nakamura, E.; Fuji, K. J. Org. Chem. 2004, 69, 7960. (j) Huang, H.; Liu, X.; Chen, H.; Zheng, Z. Tetrahedron: Asymmetry 2005, 16, 693.

14. (a) Gladiali, S.; Dore, A.; Fabbri, D.; De Lucchi, O.; Manassero, M. Tetrahedron: Asymmetry 1994, 5, 511. (b) Charruault, L.; Michelet, V.; Taras, R.; Gladiali, S.; Genet, J.-P. Chem. Commun. 2004, 850. (c) Zanoni, G.; Gladiali, S.; Marchetti, A.; Piccinini, P.; Tredici, I.; Vidari, G. Angew. Chem. Int. Ed. 2004, 43, 846.

15. (a) Chi, Y.; Zhang, X. Tetrahedron Lett. 2002, 43, 4849. For bidentate ligands with 4,5dihydro-3H-dinaphtho-[2,1-c;1',2'-e]phosphepine core see: (b) Tang, W.; Wang, W.; Chi, Y.; Zhang, X. Angew. Chem. Int. Ed. 2003, 42, 3506. (c) Chi, Y.; Zhou, Y.-G.; Zhang, X. J. Org. Chem. 2003, 68, 4120. (d) Xiao, D.; Zhang, X. Angew. Chem. Int. Ed. 2001, 40, 3425. (e) Xiao, D.; Zhang, Z.; Zhang, X. Org. Lett. 1999, 1, 1679.

16. (a) Junge, K.; Oehme, G.; Monsees, A.; Riermeier, T.; Dingerdissen, U.; Beller, M. Tetrahedron Lett. 2002, 43, 4977. (b) Junge, K.; Hagemann, B.; Enthaler, S.; Spannenberg, A.; Michalik, M.; Oehme, G.; Monsees, A.; Riermeier, T.; Beller, M. Tetrahedron: Asymmetry 2004, 15, 2621.

17. Enthaler, S.; Junge, K.; Hagemann, B.; Beller, M. Eur. J. Org. Chem. 2006, 2912.

18. (a) Junge, K.; Hagemann, B.; Enthaler, S.; Oehme, G.; Michalik, M.; Monsees, A.; Riermeier, T.; Dingerdissen, U.; Beller, M. Angew. Chem. 2004, 116, 5176; Angew. Chem. Int. Ed. 2004, 43, 5066. (b) Hagemann, B.; Junge, K.; Enthaler, S.; Michalik, M.; Riermeier, T.; Monsees, A.; Beller, M. Adv. Synth. Cat. 2005, 15, 1978.

19. (a) Chi, Y.; Zhou, Y.-G.; Zhang, X. J. Org. Chem. 2003, 68, 4120. (b) Chi, Y.; Zhang, X. Tetrahedron Lett. 2002, 43, 4849. (c) Xiao, D.; Zhang, X. Angew. Chem. Int. Ed. 2001, 40, 3425. (d) Xiao, D.; Zhang, Z.; Zhang, X. Org. Lett. 1999, 1, 1679.

20. (a) Yan, M.; Yang, L.-W.; Wong, K.-Y.; Chan, A. S. C. Chem. Commun. 1999, 11. (b) Yan, M.; Zhou, Z.-Y.; Chan, A. S. C. Chem. Commun. 2000, 115. (c) Su, L.; Li, X.; Chan, W. L.; Jia, X.; Chan, A. S. C. Tetrahedron: Asymmetry 2003, 14, 1865. 
21. Junge, K.; Oehme, G.; Monsees, A.; Riermeier, T.; Dingerdissen, U.; Beller, M. J. Organomet. Chem. 2003, 675, 91.

22. Baker, M. J.; Pringle, P. G. Chem. Commun. 1991, 1292.

23. Cserepi-Szucs, S.; Huttner, G.; Zsolnai, L.; Szolosy, A.; Hegedus, C.; Bakos, J. Inorg. Chim. Acta 1999, 296, 222.

24. Yan, M.; Li, X.; Chan, A. S. Chin. Sc. Bull. 2003, 48, 2188.

25. Sturla, S. J.; Buchwald, S. L. J. Org. Chem. 2002, 67, 3398.

26. Chapman, C. J.; Wadsworth, K. J.; Frost, C. G. J. Organomet. Chem. 2003, 680, 206.

27. Bitterer, F.; Herd, O.; Kühnel, M.; Stelzer, O.; Weferling, N.; Sheldrick, W. S.; Hahn, J.; Nagel, S.; Rösch, N. Inorg. Chem. 1998, 37, 6406.

28. For the synthesis of building block A see: (a) Arnold, L. A.; Imbos, R.; Mandoli, A.; de Vries, A. H. M.; Naasz, R.; Feringa, B. L. Tetrahedron 2000, 56, 2865. (b) Kranich, R.; Eis, K.; Geis, O.; Mühle, S.; Bats, J. W.; Schmalz, H.-G. Chem. Eur. J. 2000, 15, 2874.

29. Segupta, S.; Leite, M.; Raslan, D. S.; Quesnelle, C.; Snieckus, V. J. Org. Chem. 1992, 57, 4066.

30. Engelhardt, L. M.; Leung, W.-P.; Raston, C. L.; Salem, G.; Twiss, P.; White, A. H. J. Chem. Soc. Dalton Trans. 1988, 2403. 